\title{
COMPETITIVIDAD DE EMPRESAS TURÍSTICAS: UN ANÁLISIS DESDE EL ENFOQUE SISTÉMICO*
}

\author{
MERCY LOLLY NARVÁEZ CASTRO"*; GLADYS FERNÁNDEZ DE HURTADO** \\ \& ARACELY TERESA HENRÍQUEZ BARRÁEZ"**" \\ UNIVERSIDAD DEL ZULIA (VENEZUELA)
}

Recibido/ Received/Recebido: 09/04/2012 - Aceptado/ Accepted/Aprovado: 23/04/2013

\begin{abstract}
Resumen
Este artículo muestra los resultados de una investigación cuyo objeto fue el análisis de la competitividad de las empresas que conforman el sector de alojamiento turístico en el contexto de la localidad de la península de Paraguaná -Estado Falcón (Venezuela) bajo los lineamientos del enfoque sistémico. La metodología combina los diseños documental y de campo, dispuestos como estrategias de recolección de información. La fase de campo fue de tipo no experimental, de naturaleza transversal o transeccional, empleándose para la recolección de información una encuesta, la cual fue aplicada a un censo poblacional de nueve (9) empresas hoteleras. Como aporte se presenta una propuesta estratégica orientada no solo a alcanzar el posicionamiento competitivo del sector turístico hotelero, sino también el desarrollo de la localidad paraguanera, la cual es considerada como un destino turístico emergente a escala nacional.
\end{abstract}

Palabras clave: Competitividad sistémica, Empresas de alojamiento, Desarrollo local, Sector turístico.

\section{COMPETITIVENESS OF TOURIST COMPANIES: AN ANALYSIS FROM THE SYSTEMIC APPROACH}

\begin{abstract}
This article shows the results of a research which purpose was to analyze the competitiveness of companies from the tourism lodging sector in the context of the Peninsula de Paraguaná town Estado Falcón (Venezuela) under the guidelines of the systemic approach. The methodology combines documental and field designs, set as information collecting strategies. The non-experimental field phase had a transversal or transactional nature, used to collect information through a survey, which was applied to a census of nine (9) lodging companies. As a contribution, the article presents a strategic proposal aimed not only to reach the competitive positioning of the lodging tourist sector but also the development of the Paraguanera town, which is considered as an emerging tourist destination at national level.
\end{abstract}

Keywords: Systemic competitiveness, Hosting companies, Local development, Tourism.

* Este artículo forma parte de los resultados obtenidos del Proyecto de Investigación Científica titulado: "Competitividad de la Península de Paraguaná como destino turístico en el marco del desarrollo sostenible", adscrito a la Línea de Investigación: "Gerencia Organizacional y Competitividad Empresarial”. Financiado por el Consejo de Desarrollo Científico, Humanístico y Tecnológico de la Universidad del Zulia (LUZ) Venezuela. Fecha de Culminación del Proyecto: Mayo 2012.

** Miembro Titular del Personal Docente y de Investigación de la Universidad del Zulia (LUZ) - Venezuela. Licenciada en Administración (LUZ). Magíster en Gerencia de Empresas (LUZ). Doctora en Ciencias Gerenciales (URBE). Post-Doctora en Ciencias Gerenciales (UNEFA).Correo electrónico: romenarvaez@cantv.net, mercynarvaez@gmail.com

**:* Miembro Titular del Personal Docente y de Investigación de la Universidad del Zulia (LUZ) - Venezuela. Licenciada en Contaduría Pública (LUZ). Magíster en Gerencia de Empresas (LUZ). Doctora en Planificación y Gestión del Desarrollo Regional (LUZ). Correo electrónico: gladysfernandez2@gmail.com

**:** Miembro del Personal Docente y de Investigación de la Universidad Nacional Experimental Francisco de Miranda (UNEFM) - Venezuela. Ingeniero Industrial (UNEFM). Magíster en Gerencia de Empresas (LUZ). Correo electrónico: henriquezarat@hotmail.com 


\title{
COMPETITIVIDADE DE EMPRESAS TURÍSTICAS: UMA ANÁLISE DESDE O ENFOQUE SISTÊMICO
}

\section{Resumo}

\begin{abstract}
Este artigo mostra os resultados de uma pesquisa cujo objeto foi a análise da competitividade das empresas que formam o setor de hospedagem turística no contexto da localidade da península de Paraguaná - Estado Falcón (Venezuela) sob os lineamentos do enfoque sistêmico. A metodologia combina os desenhos documentais e de campo, dispostos como estratégias de coleta de informação. A fase de campo foi de tipo não experimental, de natureza transversal ou transeccional, empregando-se para a coleta de dados um questionário, o qual foi aplicado a um censo populacional de nove (9) empresas hoteleiras. Apresenta-se uma proposta estratégica orientada não só a atingir o posicionamento competitivo do setor turístico hoteleiro, mas também o desenvolvimento da localidade paraguanera, a qual é considerada um destino turístico emergente de escala nacional. Palavras chave: Competitividade sistêmica, Empresas de hospedagem, Desenvolvimento local, Setor turístico.
\end{abstract}

Narváez, M., Fernandez, G. \& Henríquez, A. (2013) Competitividad de empresas turísticas. Un análisis desde el enfoque sistémico. En: Revista de la Facultad de Ciencias Económicas de la Universidad Militar Nueva Granada. rev.fac.cienc.econ, XXI (1)

JEL: M19, M29.

\section{Introducción}

Toda estrategia de desarrollo de cualquier nación en la era global debe tener como pilares pactos sociales sólidos y democráticos que garanticen la estabilidad política, sistemas locales no discrecionales y formas de comportamiento de los agentes que confieren seguridad en los contratos, y la constitución de una burocracia estatal imparcial y eficiente. En tal sentido, las estrategias que adopten los países hoy en día deben tener como mínimo los siguientes elementos: políticas macroeconómicas destinadas a reducir la vulnerabilidad económica y facilitar la inversión productiva, estrategias dirigidas a desarrollar la competitividad sistémica, un enérgico reconocimiento de las prioridades de la agenda ambiental y políticas sociales muy activas, especialmente de educación, empleo y protección social (CEPAL, 2000).

En el marco de referencia antes descrito, la formulación de guías de acción para estimular la creación de factores que se orienten a alcanzar la competiti- vidad sistémica en los países, las regiones o en los sectores industriales o empresariales se constituyen en un componente activo y dinámico para alcanzar ventajas competitivas y por consiguiente en un elemento importante para promover el desarrollo en la instancia o el lugar donde se implementen.

El enfoque teórico de la competitividad sistémica tiene como premisa la integración social, a diferentes niveles, exigiendo no sólo reformas económicas, sino también un proyecto de transformación de la sociedad. La tarea pendiente para muchos países en desarrollo y en transformación radica en superar la fragmentación social y mejorar la capacidad de aprendizaje, sobre todo la capacidad para responder con prontitud y eficacia a los requerimientos de ajuste. La formación de estructuras al nivel de sociedad, como complemento de la formación de estructuras a nivel económico, eleva la capacidad de los diferentes grupos de actores para articular sus intereses y satisfacer entre todos los requerimientos tecnológico-organizativos, sociales y ambientales, entre otros (Ferrer \& Vásquez, 2005, 169). 
A la luz de estos planteamientos surge el presente artículo, el cual tiene como propósito analizar las empresas que conforman el sector de alojamiento turístico en el contexto de la localidad de la península de Paraguaná - Estado Falcón (Venezuela) bajo los lineamientos del enfoque de "competitividad sistémica". Todo ello con la finalidad de identificar los factores determinantes que influyen en el desarrollo competitivo de dicho sector y que a la vez tienen incidencia en la localidad donde están asentadas las empresas objeto de esta indagación, la cual es considerada como un destino turístico emergente a escala nacional e internacional, luego de su declaratoria como Zona Libre de Interés Turístico y Comercial en el año 1998.

Adicionalmente, a manera de propuesta se presentan algunos lineamientos estratégicos de carácter general para la promoción y desarrollo local de la Península de Paraguaná (espacio geográfico donde se desarrolló la investigación), sobre la base del análisis de la competitividad sistémica en el sector empresarial de alojamiento turístico.

\section{Metodología}

En este artículo se emplearon criterios teóricos y empíricos que permitieron analizar desde una perspectiva sistémica, el comportamiento de los factores de competitividad vinculados al sector empresarial dedicado a la prestación del servicio turístico de hospedaje (empresas categorizadas como hoteles), ubicado en la península de Paraguaná, punto más septentrional de Venezuela y América del Sur, catalogado como destino turístico emergente, con un particular significado estratégico económico, social, cultural y geopolítico, tanto para el país como para la región y en el cual convergen fuerzas de carácter endógeno capaces de estimular el desarrollo local.

En tal sentido, este trabajo se estructura en dos partes; la primera expone una disertación gene- ral sobre las teorías vinculadas al turismo y a los elementos conceptuales del enfoque sistémico de la competitividad. La segunda declara la metódica seguida a través de la combinación de diseños documental y de campo, dispuestos como estrategias de recolección de información. El diseño de campo se realizó con base en la aplicación de un cuestionario estructurado, de aplicación única (transeccional o transversal).

La población estuvo constituida por doce (12) empresas hoteleras registradas en CORPOTULIPA ${ }^{1}$, en su mayoría pequeñas y medianas organizaciones que se han venido conformando luego de la declaratoria de Paraguaná como Zona Libre de Inversión Turística y Comercial en el año 1998. Sin embargo, de la totalidad de la población solo nueve (9) empresas accedieron a responder el cuestionario diseñado para la recolección de datos.

Por lo antes expuesto, cabe destacar entonces que para efectos de esta investigación se utilizó un censo poblacional, el cual es utilizado para estudiar todos los elementos de una población, y según lo expresado por Méndez $(2001,182)$, se recomienda la utilización de este cuando la población de interés sea tan pequeña que un costo y tiempo adicionales en el estudio de la misma estén plenamente justificados.

\section{El turismo como actividad para el desarrollo económico}

El turismo es un concepto de carácter complejo que aun está en construcción, pues es razón de estudio de diferentes áreas del conocimiento con las cuales se relaciona. En tal sentido, existen diferentes definiciones que resultan parciales e incompletas. Sin embargo, para efectos de este trabajo se hará énfasis en la visión de este fenómeno desde la postura del desarrollo económico, dado que el mismo produce ingresos y mejora la calidad de vida de los espacios geográficos donde tiene lugar dicha actividad.

1 Corporación para la Zona Libre para el Fomento de la Inversión Turística en la Península de Paraguaná (CORPOTULIPA). Es un ente público adscrito al Ministerio del Poder Popular para la Economía y Finanzas de la República Bolivariana de Venezuela. Dicha corporación es el órgano rector administrativo de la Zona Libre, cuya labor fundamental consiste en ser el articulador de las políticas de desarrollo turístico de la región. 
Desde la concepción económica, el turismo es definido como "(...) el concepto que comprende todos los procesos, especialmente económicos, que se manifiesta en la afluencia, permanencia y regreso del turista hacia, en y fuera de un determinado municipio, país o estado" (Fernández, 1974, 27).

Mochón $(2004,11)$ señala que la actividad económica por excelencia es la producción de una amplia gama de bienes y servicios cuyo último destino es el consumo por parte de los agentes económicos (familias o economías domesticas, las empresas y el sector público), los cuales se pueden clasificar distinguiendo tres grandes sectores (primario, secundario y terciario).

El sector primario abarca las actividades que se realizan en torno a las bases de recursos naturales, como: agrícolas, pesqueras, ganaderas y extractivas. El sector secundario recoge las actividades industriales mediante las cuales se transforman los bienes; y el sector terciario o de servicios, reúne las encaminadas a satisfacer necesidades de servicios productivos que no se plasmen en algo material.

Dentro del sector terciario o de servicios se integran el conjunto de actividades que conforman el turismo, el cual es considerado como la industria más grande del mundo, superior a la del automóvil, el acero, productos electrónicos y la agricultura. Al respecto, McIntosh et al. $(2008,20)$ señalan que el turismo ha crecido hasta convertirse en una actividad de importancia y significado mundial; en varios países, el turismo es el producto más grande en el comercio internacional, en muchos otros se clasifica entre las tres industrias más importantes. Vale decir, que el turismo ha crecido rápidamente hasta convertirse en una gran fuerza económica y social en el mundo.

\subsection{Las empresas turísticas}

Según Mochón $(2004,17)$, los principales tipos de empresas que integran el sector turístico son las siguientes:

- Empresas relacionadas con el trasporte de viajeros, comprenden las empresas aéreas, de trasporte por ferrocarril, de autobuses, de trasporte marítimo y de alquiler de autos.

- Empresas que ofrecen alojamiento, esto es las empresas hoteleras en sentido amplio; hoteles, hostales, pensiones, así como las responsables de la oferta extrahotelera; alquiler de apartamentos, casas rurales, campamentos de turismo, además de otros alojamientos específicos: residencias de tiempo libre para el turismo social, albergues juveniles, alquiler de habitaciones en casas particulares con derecho a desayuno, empresas de tiempo compartido (timesharing), entre otras.

- Empresas creadoras y comercializadoras de bienes y servicios turísticos (intermediarios turísticos), estas realizan labores de intermediación para la venta de servicios turísticos poniendo en contacto los oferentes (hoteles, compañías aéreas, entre otros) con el turista; y también crean productos turísticos (paquetes turísticos) mediante la contratación de grandes volúmenes de servicios diversos, que una vez agrupados se ofrecen al turista de forma conjunta a un precio único, siempre inferior al que resultaría si el turista contratase por sí mismo los distintos servicios que integran el paquete turístico ofertado.

- Empresas relacionadas con la restauración, las cuales se especializan en ofrecer comidas y bebidas durante el viaje y la estancia turística.

- Empresas que proveen actividades recreativas, eventos y visitas a lugares de interés turístico. Las actividades ofertadas por este tipo de empresas son muy diferentes y su nexo de unión con el turismo es el de ser demandadas por el turista. Están relacionadas con puertos deportivos, campos de golf, teatros, conciertos, festivales, parques temáticos, museos, parques nacionales, ferias comerciales, entre otros.

- Empresas especializadas en ofertar servicios periféricos o complementarios. Son servicios accesorios ofrecidos principalmente por el sector privado que amplían y complementan la oferta turística básica tanto en origen como en destino.

En este artículo se hará énfasis en el estudio de las empresas turísticas dedicadas a la prestación del 
servicio de alojamiento, específicamente las que se incluyen en la categoría de hoteles. En tal sentido, se estima necesario incluir algunos aspectos teóricos vinculados con esta categoría de empresas que cumplen con una actividad empresarial subsecuente del turismo.

Ramírez (2007, 14), plantea que la hotelería como actividad empresarial responde a varios conceptos y necesidades, a saber:

- Para la Unión Internacional de Organismos Oficiales de Turismo, es el servicio de alojamiento, alimentación y servicios complementarios requeridos por el turista que se proporcionan en un establecimiento con un mínimo de 10 habitantes.

- Para el público en general, es el lugar de descanso por un breve período, en uso de su tiempo libre.

- Para el ejecutivo y en general para cualquier persona vinculada con los negocios, es un punto de llegada con todas las facilidades y comodidades que requiere su actividad en situaciones de bienestar y seguridad, similares y mejores a las de su propio despacho profesional.

- Para cualquier individuo en particular, es un lugar de alojamiento con las mejores condiciones de bienestar y seguridad, en situaciones de carácter temporal, o cuando se trata de un modo de vida escogido de carácter permanente.

\section{Competitividad empresarial y competitividad sistémica}

\subsection{Competitividad Empresarial}

El termino competitividad se circunscribe inicialmente a la economía empresarial, y la capacidad de una empresa para ser competitiva se valora en relación a sus posibilidades de mantener o aumentar la rentabilidad de sus activos en las condiciones prevalecientes en mercados abiertos a la competencia. Es decir, una empresa es competitiva cuando es capaz de obtener suficientes beneficios mediante el uso eficiente de sus recursos y habilidades, siendo capaz de ubicar exitosamente sus productos en el mercado en condiciones de libre competencia.
Antonorsi (1999, 20) expone algunos aspectos esenciales para entender la dimensión del concepto desde la perspectiva empresarial:

- La competitividad es una capacidad de la empresa. No es un don gratuito, es una habilidad que solamente se puede lograr con esfuerzo. Es una capacidad que debe ser buscada, desarrollada y perfeccionada.

- La competitividad implica a toda la empresa y es el producto de toda ella. No solamente de su función de mercadeo. Para ser competitiva, la empresa debe ser capaz de diseñar, producir y mercadear productos y servicios competitivos; para lograrlo, toda la organización debe apoyar estas capacidades. La competitividad es una capacidad, una habilidad de la empresa en conjunto.

- La competitividad se manifiesta en mejores productos y servicios. En efecto, se compite en el mercado con productos / servicios, pero estos productos / servicios no salen de la nada, se derivan de una empresa competitiva que los hace posible y los respalda de manera sostenida.

- Implica satisfacción del cliente. Se compite por los favores de una clientela, es decir satisfaciendo a los clientes oportunamente con productos de calidad y precio adecuados, es como la empresa logra mantener y aumentar su participación en el mercado, y por tanto, sobrevivir y progresar.

- La competitividad es una cualidad vital. Si la empresa no tiene capacidad, habilidad competitiva no puede sobrevivir y menos progresar. $\mathrm{Si}$ no se tiene capacidad competitiva la empresa va perdiendo vida, poco a poco, o rápidamente, hasta morir. La competitividad es una cualidad necesaria para poder competir y de esa manera sobrevivir y progresar.

- Implica competir con rivales. Es una cualidad para competir con (contra) otros, quienes también estén buscando mejorar su competitividad. Por esa razón obvia, la competitividad es una cualidad cuyo desarrollo no tiene fin. La competitividad implica mejoras y superación continua frente a los rivales competitivos. 
Ahora bien, en la actualidad el concepto de competitividad no solo se circunscribe al ámbito de las empresas, sino que va más allá e incluye el análisis de sectores empresariales, regiones y países. Al respecto, señalan Morales \& Castellanos (2007, 118), la competitividad es un concepto que no tiene límites precisos y se define en relación con otros conceptos. La definición operativa de la competitividad depende del punto de referencia -nación, sector, firma- , del tipo de producto analizado -bienes básicos, productos diferenciados, cadenas productivas, etapas de producción- $y$ del objetivo de la indagación -corto o largo plazo, explotación de mercados, reconversión, entre otros-.

Por su parte, Rojas et al. $(2000,10)$ señalan que la medición de la competitividad implica la determinación de los componentes o factores que la generan y el grado de impacto de los mismos. En tal sentido, estos autores establecen que el desempeño competitivo de una empresa, industria o nación está condicionado por la conjugación de diversos factores: internos a la empresa, sectoriales, sistémicos y de desarrollo microeconómico.

- Factores internos a la empresa: Son los que aparecen bajo su ámbito de decisión y por medio de los cuales la empresa procura distinguirse de sus competidores. Entre ellos destacan: capacidad tecnológica y productiva, calidad de los recursos humanos, conocimiento del mercado y la capacidad de adecuarse a sus especificidades, relaciones privilegiadas con los clientes y con los abastecedores de insumos, materias primas y bienes de capital.

- Factores sectoriales: Si bien no son manejados directamente por la empresa, están parcialmente en su área de influencia. Involucran el contexto decisional de las empresas y los productores.

- Factores sistémicos: Constituyen elementos externos a la empresa productiva, afectan el entorno donde se desarrolla y pueden tener importancia variable en la definición del ambiente competitivo y en las posibilidades para construir estrategias de competitividad por parte de las empresas. Entre ellos destacan: acceso al financiamiento y la infraestructura tecnológica e institucional.
- Factores de desarrollo microeconómico: A partir de los cambios tecnológicos emergentes surge un nuevo formato organizacional que conlleva la descentralización de las decisiones y una creciente participación de la fuerza laboral en las decisiones y ganancias de la empresa, al tiempo que permea las relaciones en y entre las empresas. Algunos de estos factores son: la capacitación y gestión tecnológica de las empresas, los nuevos esquemas organizacionales, estrategias de venta y de relaciones con los proveedores y recursos humanos.

\subsection{Elementos conceptuales del enfoque sistémico de la competitividad}

Las estrategias de desarrollo de las regiones o localidades deben orientarse, entre muchos otros aspectos, a la transformación dinámica de las estructuras productivas y sociales mediante la interrelación de los sectores público, privado y la comunidad en general, movilizando y canalizando los recursos y capacidades del territorio, lo cual permitirá solventar las deficiencias que les afectan y alcanzar objetivos comunes a través del esfuerzo conjunto.

De manera complementaria con el anterior planteamiento Boisier $(2004,54)$ afirma que el desarrollo local hay que entenderlo como un fenómeno que se presenta en por lo menos cuatro planos que se entrelazan entre sí: en primer lugar, se refiere o se manifiesta en el plano político, como una creciente capacidad local de diseñar y ejecutar políticas de desarrollo, y sobre todo, la capacidad de negociar con los elementos que definen el entorno del territorio. En segundo lugar, se manifiesta en el plano económico, y se refiere a la apropiación y reinversión local de parte del excedente a fin de diversificar la economía local, dándole al mismo tiempo una base permanente de sustentación en el largo plazo. Como tercer plano, el desarrollo local es interpretado en el plano científico y tecnológico como la capacidad interna de un sistema - en este caso, de un territorio organizado- para generar sus propios impulsos tecnológicos de cambio, capaces de provocar modificaciones cualitativas en el sistema mismo; y finalmente se plantea en el plano de la cultura, como una matriz generadora de la identidad 
socio-territorial, lo cual es fundamental para considerar un verdadero proceso de desarrollo.

Por su parte, Narváez et al. $(2008,76)$ expresan que el desarrollo local parte de la capacidad para la integración de visiones e intereses y la concertación de agentes públicos y privados con incidencia en el territorio. Así pues, el desarrollo debe ser visto como el resultado que se genera del esfuerzo organizado del conjunto de la comunidad y muy particularmente, para el aspecto económico, de la capacidad de conformar algo más que una simple aglomeración de empresas por industrias o sectores, donde se adopten nuevos modelos de organización que impulsen la flexibilidad y la descentralización operativa, favoreciendo la constitución de unidades estratégicas de actuación o de negocios $y$ donde se priorizan acciones colectivas sobre las individuales, a través de la conformación de redes que promuevan la dinamización empresarial, el estímulo a las innovaciones y la utilización más eficaz posible de todo el potencial de desarrollo disponible o incorporable al territorio.

Adicionalmente, es necesario resaltar que este enfoque de desarrollo también incluye el papel del Estado, el cual adquiere otras posibilidades de expresión. Al respecto, Madoery $(2000,4)$ señala que el Estado-Nación está sometido a fuertes tensiones "desde arriba", por la consolidación de ámbitos institucionales supranacionales y "desde abajo" por la afirmación de territorios subnacionales más visibles y protagónicos. Es por ello, que las políticas del gobierno central descansan sobre las dinámicas de las comunidades donde las empresas, las autoridades públicas, y la sociedad civil pueden establecer nuevos compromisos articulando en el territorio los diversos planos de lo económico, urbano, social, medioambiental, cultural e institucional, como componentes de la política de desarrollo local.

Ahora bien, el concepto de competitividad sistémica como planteamiento teórico referido al desarrollo parte de la premisa de que éste es producto de un patrón de interacción compleja y dinámica entre el Estado, las empresas, las instituciones intermediarias y la capacidad organizativa de una sociedad. Di- cho concepto surge como consecuencia de los trabajos empíricos realizados alrededor de los años 90 por la Comisión Económica para América Latina y el Caribe (CEPAL), sobre diversos países en vías de desarrollo y además, de la visión compleja que plantea un marco de análisis integrado para la discusión de aspectos económicos y sociales referidos a los factores determinantes de la competitividad.

En opinión de Esser et al. (2001, 146), las ideasfuerza que sustentan este concepto son: (a) la competitividad industrial duradera no surge solamente a través de la estabilización de las condiciones macroeconómicas y la creación de las correspondientes estructuras incentivadoras. Si bien una sólida política macroeconómica es necesaria, no es suficiente para el logro del desarrollo industrial; (b) el éxito del desarrollo industrial apoya y multiplica los esfuerzos de las empresas individuales o los conglomerados (cluster); y (c) la creación de un entorno eficiente es una tarea que no incumbe exclusivamente al Estado, sino que para su consecución juegan un papel importante, los diferentes actores no estatales (empresas, asociaciones, sindicatos y comunidad, entre otros).

Por otra parte, el concepto de competitividad sistémica se fundamenta en tres elementos, a saber: el primero es el papel del conocimiento como pilar de la competitividad, ya que este factor es el único que permite producir bienes y servicios que pasen la prueba de los mercados internacionales, además de proteger e incrementar los ingresos reales de los ciudadanos. El segundo, es la insistencia en que, más allá de los esfuerzos aislados de las empresas, la competitividad es una consecuencia de todo un sector, de su interacción con otros que le suministran bienes y servicios y, en última instancia, del buen desempeño de todo el sistema económico. El tercero es el reconocimiento de que el desarrollo de buenos mercados de tecnología y de encadenamientos productivos depende de los esfuerzos conscientes del Estado, de las asociaciones empresariales y especialmente de las formas de colaboración entre el Estado y el sector privado con el objeto de crear el círculo virtuoso sobre el que se sustenta la competitividad (CEPAL, 2003, 147). 
Adicionalmente, CEPAL (2001) plantea que los elementos distintivos del concepto de competitividad sistémica son: (a) la diferenciación de cuatro niveles analíticos (meta, macro, meso y micro) y (b) la vinculación de los elementos de cuatro diferentes escuelas del pensamiento: la economía de la innovación y de las teorías evolutivas; la escuela post estructuralista con la redefinición del papel del Estado en los procesos de industrialización tardía, la nueva economía institucional y la escuela moderna de administración. En el área de las ciencias sociales, la competitividad sistémica se relaciona con la sociología industrial, la geografía económica y las ciencias políticas, todas ellas fundamentadas en la noción de redes, elemento central del concepto de competitividad sistémica.

\subsubsection{Niveles analíticos de la competitividad sistémica}

De acuerdo con la CEPAL (2001, 7), los cuatro niveles analíticos de competitividad sistémica, son:

- Nivel Meta: Gobernabilidad y competitividad industrial. Constituido por los patrones de organización política y económica orientados al desarrollo y por la estructura competitiva de la economía en su conjunto.

- Nivel Macro: Vinculación por la estabilización económica y la liberalización con la capacidad de transformación. Compuesto por el conjunto de condiciones macroeconómicas estables, particularmente una política cambiaria realista y una política comercial que estimule la industria local.

- Nivel Meso: Apoyo a los esfuerzos de las empresas. Formado por las políticas específicas para la creación de ventajas competitivas, por el entorno y por las instituciones.

- Nivel Micro: Requerimientos tecnológicos e institucionales. Constituido por la capacidad individual de desarrollar procesos de mejora continua y asociaciones, así como redes de empresa con fuertes externalidades.
$\mathrm{Al}$ respecto de estos niveles analíticos cabe destacar que la competitividad sistémica se caracteriza y distingue por reconocer que un desarrollo industrial exitoso no se logra meramente a través de una función de producción en el nivel micro, o de condiciones económicas estables en el nivel macro, sino también por la existencia de medidas del gobierno y organizaciones privadas de desarrollo orientadas a fortalecer la competitividad de las empresas (nivel meso). Además, la capacidad de vincular las políticas meso y macro dependen de un conjunto de estructuras políticas y económicas y de un conjunto de factores socioculturales y patrones básicos de organización (nivel meta) (Esser et al.,1996).

\section{Caracterización de la localidad donde se contextualiza el estudio}

La Península de Paraguaná está situada al norte del Estado Falcón - Venezuela frente al Mar Caribe, presentando una excelente ubicación por su cercanía a las Islas del Caribe y su facilidad de acceso tanto por vía terrestre como aérea. Tal posición le permite ser puerta abierta para el desarrollo de la actividad comercial y turística, además de tener cercanía a ciertos mercados, con los cuales puede realizar actividades de intercambio y fortalecer su mercado.

Adicionalmente, a partir del año 1998 se crea para la Península de Paraguaná la Ley de Zona Libre para la Inversión Turística (puesta en vigencia en febrero de 1999). Esta ley tiene por objeto establecer un régimen especial territorial de carácter fiscal para el fomento de la prestación de servicios en la actividad turística y comercial conexa al turismo, la cual abarca el área geográfica comprendida por los territorios de los Municipios Carirubana, Falcón y Los Taques del Estado Falcón. (Artículo.1 de la Ley de Zona Libre, 1998).

Esta Ley, aunada a los sitios naturales, culturales e históricos que presenta la localidad, ha dado paso al auge de la actividad turística en la península. Es por ello que la Fundación para el Desarrollo de la Región Centro Occidental de Venezuela (FUDECO) ${ }^{2}$

2 Fundación para el Desarrollo de la Región Centro Occidental de Venezuela. FUDECO (1998). (Disponible: http://www.fudeco. org). 
en el año 1996 diseñó ocho (8) productos turísticos para la península, a saber: Playas del Este, Playas del Oeste, Bahías del Norte, Ecocircuitos, Media Luna de Pueblos y Hatos, Balcón del Caribe, Guaranao de Cruceros y Punto Fijo de Negocios. Dichos productos fueron integrados en el "Modelo de Desarrollo Turístico Sustentable de la Península de Paraguaná", tomando como referencia la oferta del Caribe, en particular Aruba, Bonaire, Curazao y la Isla de Margarita, potenciando a Paraguaná como puerta de entrada del turismo internacional en el Occidente de Venezuela.

La implementación de la Ley de Zona Libre, ha permitido que la península se posicione en el ámbito nacional con una imagen de destino turístico. Evidenciándose una marcada afluencia de visitantes en las temporadas de Diciembre, Carnaval, Semana Santa y Agosto, así como también durante fines de semana y días feriados. Además, la Zona Libre ha permitido mejorar, en algunos aspectos la calidad de vida de los residentes de Paraguaná, y su consolidación más allá de representar un simple régimen fiscal, significa el desarrollo futuro de sus pobladores y la diversificación de la economía de la localidad en general, la cual siempre había estado concentrada en actividades petroleras y dependientes del sector público.

Como resultado de la diversificación económica a través del impulso de la actividad turística y comercial en la localidad se evidencia el establecimiento de empresas e instituciones dedicadas a las siguientes labores: servicios de transporte, alimentos, alojamiento en sus diversas modalidades, artesanía local, servicios recreativos, empresas comerciales, entre otros. Así mismo, han sido ejecutadas nuevas obras de infraestructura de carácter público y privado que redundan en mayor bienestar y calidad de vida de los residentes.

\section{Discusión y análisis}

Los resultados expuestos en este aparte del artículo pretenden presentar un diagnóstico general que describe algunas dimensiones comprendidas en los niveles analíticos establecidos en el enfoque teórico de la competitividad sistémica, a partir de la postura de los gerentes y empresarios de las organizaciones que conforman el sector hotelero de la península de Paraguaná.

\subsection{Nivel Micro}

Fea $(1995,40)$ señala que una empresa es competitiva cuando logra maximizar la calidad de cada uno de estos factores:

- Un proceso productivo óptimo, es decir, la mejor organización tecnológica para producir.

- Recursos excelentes, porque dispone de un genio empresarial superior, de un factor humano motivado, profesionalmente preparado y con un notable bagaje cognoscitivo; de estructuras sociales eficientes, de capitales adecuados y de óptimos materiales.

- Elevada calidad del proceso de transformación, con productos y servicios capaces de ofrecer al cliente la máxima satisfacción, en términos de presentaciones, fiabilidad, precios y servicio.

- Considerable generación de valor añadido, al optimizar la utilización de los factores anteriores, los recursos originan una producción sobresaliente por calidad y cantidad.

- Destacada calidad de productos y servicios y minimización del coste, que permiten un margen elevado entre los recursos empleados y el precio de venta.

Para efectos de este artículo se consideraron como rasgos o factores competitivos a nivel micro sistémico los siguientes: recursos, infraestructura, actividades, procesos, calidad de servicio y satisfacción al cliente. Así pues, al analizar los rasgos o factores competitivos que caracterizan a las empresas hoteleras objeto de este estudio se evidencia en la Tabla 1.

En lo correspondiente a los recursos de las empresas, los encuestados manifestaron en alto $(77,78 \%)$ 
y mediano grado $(22,22 \%)$ que conforman un equipo empresarial capaz, creativo y con espíritu emprendedor. Además, un significativo porcentaje de ellos $(66,67 \%)$, cuenta con un recurso humano motivado, profesionalmente preparado y poseedor de un consolidado capital intelectual. Ahora bien, en cuanto al capital económico necesario para el desenvolvimiento normal de las operaciones de la empresa se evidencia, de las respuestas de los entrevistados, que cuentan en mediano $(55,55 \%)$ y alto grado $(33,33 \%)$ con suficientes recursos para emprender procesos de mejora y crecimiento. Y respecto a la estructura organizacional, solo el $55.55 \%$ de ellos considera que cuenta con una estructura flexible que le permite adaptarse a los cambios del entorno interno y externo.

Tabla 1. Rasgos de Competitividad (Nivel Micro) ${ }^{3}$

\begin{tabular}{|c|c|c|c|c|}
\hline Rasgos de Competitividad (Nivel Micro) & $\begin{array}{l}\text { Bajo } \\
\text { Grado }\end{array}$ & $\begin{array}{l}\text { Mediano } \\
\text { Grado }\end{array}$ & $\begin{array}{l}\text { Alto } \\
\text { Grado }\end{array}$ & NA \\
\hline \multicolumn{5}{|c|}{ (Expresado en términos porcentuales) } \\
\hline 1. La empresa dispone de un equipo empresarial capaz, creativo y con espíritu emprendedor & & 22.22 & 77.78 & \\
\hline $\begin{array}{l}\text { 2. La empresa posee un recurso humano motivado, profesionalmente preparado y poseedor de un con- } \\
\text { solidado capital intelectual }\end{array}$ & & 33.33 & 66.67 & \\
\hline $\begin{array}{l}\text { 3. La empresa cuenta con una estructura organizacional flexible que le permita adaptarse a los cambios } \\
\text { del entorno interno y externo }\end{array}$ & 22,22 & 55.55 & 22.22 & \\
\hline 4. La empresa cuenta con el capital suficiente para emprender procesos de mejora y crecimiento & & 55.55 & 33.33 & 11.11 \\
\hline $\begin{array}{l}\text { 5. La empresa cuenta con infraestructura física básica adecuada a las necesidades de turistas nacionales } \\
\text { y extranjeros }\end{array}$ & & 22,22 & 77.78 & \\
\hline $\begin{array}{l}\text { 6. La empresa posee infraestructura complementaria que permite satisfacer necesidades de los turistas } \\
\text { que se hospedan en la misma. }\end{array}$ & 33,33 & 66,67 & & \\
\hline \multicolumn{5}{|l|}{ Actividades y Procesos } \\
\hline $\begin{array}{l}\text { 7. El proceso de prestación de servicios de la empresa es coherente con los objetivos organizacionales, } \\
\text { la misión y la visión establecidos por la misma. }\end{array}$ & & & 88.88 & 11.11 \\
\hline $\begin{array}{l}\text { 8. La empresa tiene identificados los procesos claves (procesos implicados en la creación del producto } \\
\text { o servicio) y de apoyo (sustentan los procesos clave, proporcionan insumos, tecnología, recurso hu- } \\
\text { mano) que componen la cadena de valor }\end{array}$ & & 33.33 & 66.67 & \\
\hline 9. La empresa dispone de información sobre el desempeño de sus procesos clave y de apoyo & & 33.33 & 66.67 & \\
\hline \multicolumn{5}{|l|}{ Calidad de Servicio } \\
\hline $\begin{array}{l}\text { 10. La empresa cuenta con herramientas y metodologías para determinar si la oferta de servicios que } \\
\text { brinda está de acuerdo a la demanda y tendencias del mercado }\end{array}$ & & 33.33 & 55.55 & 11.11 \\
\hline 11. La empresa promueve el desarrollo e innovación en los servicios que ofrece & 11.11 & 11.11 & 77.78 & \\
\hline \multicolumn{5}{|l|}{ Satisfacción del Cliente } \\
\hline $\begin{array}{l}\text { 12. La empresa conoce las necesidades y prioridades que el cliente requiere atendiendo posibles } \\
\text { acciones de mejora en función de éstas }\end{array}$ & & & 100 & \\
\hline $\begin{array}{l}\text { 13. La empresa proporciona a sus clientes además de lo que necesita otros beneficios o servicios tales } \\
\text { como: servicio de trasporte, guía turística, restauración, entre otros }\end{array}$ & & 33.33 & 66.67 & \\
\hline 14. La empresa conoce cómo son evaluados por sus clientes los servicios que ofrece & & 22.22 & 77.78 & \\
\hline $\begin{array}{l}\text { 15. La empresa se evalúa constantemente con el objeto de identificar los factores que debe mejorar para } \\
\text { incrementar el nivel de satisfacción al cliente y la penetración en el mercado }\end{array}$ & 22.22 & 77.78 & & \\
\hline
\end{tabular}

3 Fuente: Elaboración propia a partir de instrumento de recolección de datos del año 2011. 
En lo referente a infraestructura física básica y complementaria para la prestación del servicio de hotelería se evidencia, de las respuestas obtenidas de los gerentes encuestados, que muchas de las empresas hoteleras cuentan con infraestructura básica $(77,78 \%)$ para cumplir con el servicio de alojamiento. No obstante, solo el 66,67\% de estas dispone de instalaciones para la prestación de servicios complementarios requeridos por el turista.

En relación a las actividades y procesos se puede apreciar en un alto porcentaje (88,88\%), que la prestación de los servicios de las empresas es coherente con los objetivos organizacionales, la misión y la visión establecidos. Asimismo, dichas organizaciones tiene identificados tanto los procesos claves (procesos medulares implicados en la prestación del servicio de hotelería), como los procesos de apoyo que sustentan los procesos clave y que componen la cadena de valor. Adicionalmente, poseen información sobre el desempeño de dichos procesos.

Con respecto a la calidad del servicio prestado por las empresas, los encuestados manifiestan en mediano y alto grado $(33,33 \%$ y $55,55 \%$ respectivamente), que poseen herramientas y metodologías para determinar si la oferta de servicios que brindan está de acuerdo a la demanda y tendencias del mercado. Igualmente, un $77,78 \%$ de los consultados considera que promueve el desarrollo e innovación en los servicios que ofrece.

En relación a la satisfacción de los turistas que visitan las empresas hoteleras objeto de estudio, el $100 \%$ de los gerentes manifiesta que conoce las necesidades y prioridades que el cliente demanda, atendiendo posibles acciones de mejora en función de éstas. Además, el 77,78\% de estos conoce cómo son evaluados por sus clientes los servicios que ofrece. Sin embargo, solo el $66,67 \%$ de las empresas proporcionan a sus usuarios otros beneficios tales como: servicio de trasporte, guía turística, restauración, entre otros.

Del análisis de lo antes descrito se infiere que aun cuando las empresas objeto de esta investigación cuentan con importantes rasgos micro sistémicos que se pueden constituir en ventajas competitivas, también poseen ciertas limitaciones que las hace menos calificadas para convertirse en organizaciones líderes que sean capaces de competir con otras empresas ubicadas no solo en la misma localidad sino también con otras situadas en destinos turísticos tanto a nivel local, nacional e internacional.

\subsection{Nivel Macro}

El verdadero reto de cualquier país en el mundo es lograr alcanzar el crecimiento económico sostenido, pero para ello se deben crear las condiciones que permitan que las personas, las empresas, los sectores industriales y por ende, el país sean productivos y competitivos. Para efectos de este análisis se presenta una breve reseña que comprende algunos aspectos de las condiciones económicas del país en los últimos años.

Durante los últimos cuarenta años, Venezuela ha estado permanentemente en crisis económica. La marcada fluctuación de los precios del petróleo (principal producto de exportación a nivel nacional) a partir de 1970, ha creado una fuerte volatilidad de los ingresos fiscales. Esta variabilidad en el recaudo fiscal ha dejado una secuela de desequilibrios y distorsiones en la economía (Penfold et al., 2009, 27).

Haciendo referencia a la situación cambiaria desde el año 1983 hasta el presente, Venezuela ha tenido más de ocho regímenes cambiarios distintos, tres de los cuales han implicado el control de cambio administrado por el Gobierno. A pesar de tales controles, la moneda venezolana se ha devaluado en términos nominales en más de un $50.000 \%$ durante este período. Las sucesivas devaluaciones de la moneda a partir de 1983 han ocasionado severas pérdidas en el ahorro nacional, empobrecido a la población menos protegida y contribuido a generar un sostenido proceso inflacionario.

A partir del año 1998 ha habido un significativo aumento en los precios del petróleo, lo cual ha traído un vertiginoso crecimiento del ingreso petrolero nacional y, en consecuencia, un importante aumento del gasto público que ha ocasionado un incremento de la moneda circulante e impulso de la inflación. En opinión de Penfold et al. $(2009,29)$, además de 
la inflación y fuerte crecimiento en el gasto público se han aplicado políticas que redundan en aumentos directos de los costos de operación de las empresas. Es más, estas políticas han debilitado la capacidad de las empresas de reorientar en forma expedita sus recursos humanos y otras capacidades hacia productos o servicios que fortalecieran su posición competitiva. Adicionalmente, los últimos diez años han estado marcados por la alta inseguridad jurídica respecto a la propiedad privada, lo cual ha producido un ambiente de exacerbada confrontación entre el gobierno y el sector privado.

Los autores antes mencionados también indican que las políticas gubernamentales de este período relacionadas con las variables macroeconómicas han generado importantes impactos, entre los que se destacan: incremento exponencial de las importaciones, en razón de que el control de cambio y la regulación de precios hace más rentable esta actividad que la producción local; altos índices inflacionarios; y producto de todo lo antes mencionado, ha aumentado notablemente la salida de capitales, lo que evidencia desconfianza en el futuro de Venezuela. Esta salida de capitales ha aumentado como consecuencia de la percepción del cierre de opciones de inversión, producto de la nacionalización de diversas industrias.

Ahora bien, en lo correspondiente al comportamiento del turismo como actividad económica en Venezuela, vale decir, que a pesar de ser un país primario exportador, ha considerado dentro de sus planes económicos la actividad turística como una prioridad, pues posee inmensas potencialidades naturales, algunas únicas en el mundo, tales como: extensiones de costa en el Mar Caribe, importantes montañas andinas, bosques tropicales amazónicos, extensas llanuras habitadas por diversidad de fauna exótica y los famosos tepuyes, las formaciones rocosas más elevadas y antiguas en el mundo; que le han permitido desarrollar destinos turísticos de larga tradición como lo son: Mérida en el Estado Mérida, la Isla de Margarita en el Estado Nueva Esparta, la Gran Sabana en el Estado Bolívar y el Archipiélago de Los Roques en el Estado Vargas.

Sin embargo, la contribución de la actividad turística en Venezuela es muy pequeña en comparación con la actividad petrolera y manufacturera. Según señalan Villamediana \& Berbesi $(2009$, 9) en el sistema de cuentas nacionales no existe la actividad turismo para indagar sobre su contribución al Producto Interno Bruto (PIB) Venezolano, pues en los datos del Instituto Nacional de Estadística la actividad hoteles y restaurantes (que se utiliza como aproximación al turismo) es de menos del $2 \%$.

Por otra parte, indican los precitados autores que en comparación con otros países de América Latina, Venezuela ocupa el penúltimo lugar de la región en cuanto al número de personas no residentes que viajan a Venezuela (turismo receptivo) y en lo referente al turismo interno (movilización de los residentes del país) se evidencia una tendencia favorable con una variación positiva interanual de 90\%, que aún cuando es significativa está condicionada por la experiencia de los turistas, la cual en muchos casos se ve perjudicada por factores tales como: malos servicios, molestias, congestionamiento, contaminación, inseguridad y hostilidad de residentes o autoridades. En correspondencia a la anterior afirmación, Francés (2003, $60)$ indica que Venezuela cuenta con escasa tradición como país receptor de turistas, a pesar de su notable dotación de atracciones naturales y culturales. Según este autor, el desarrollo turístico en Venezuela ha respondido fundamentalmente a la demanda nacional y a la demanda extranjera de negocios.

Ahora bien, haciendo especial énfasis a la localidad donde se circunscribe este estudio, cabe señalar que con respecto al porcentaje de visitantes del destino a nivel nacional e internacional, se destaca que la mayor proporción está dada por la afluencia de turistas y visitantes nacionales; según información presentada por el Consejo Nacional de Promoción de Inversiones (CONAPRI, 2008) la procedencia específica de los mismos se corresponde con los estados Zulia, Distrito Capital, Mérida, Carabobo, Lara, Táchira y Aragua. Adicionalmente, resalta la escasa afluencia turística de visitantes extranjeros a la península de Paraguaná, lo que se traduce en que la misma no es considerada aún como un destino turístico competitivo a escala internacional.

Todo lo antes mencionado permite inferir que en Venezuela es importante y pertinente promover ini- 
ciativas que impulsen y mejoren el turismo como actividad económica, pues aun cuando el país cuenta con significativas ventajas comparativas requiere de la creación de infraestructuras y políticas gubernamentales que permitan estimular e impulsar esta importante actividad y, consecuentemente, el fortalecimiento del territorio como espacio de desarrollo.

\subsection{Nivel Meso}

Este nivel de análisis de la competitividad sistémica indica la existencia de medidas específicas del gobierno y de organizaciones privadas para el desarrollo orientadas a fortalecer la competitividad de las empresas. Según señalan Esser et al. (2001,150), la estructuración del mesonivel trata del desenvolvimiento de un conjunto de instituciones eficaces y de la capacidad de interacción estrecha entre los actores públicos y privados de un conglomerado.

La formulación de políticas de competitividad en este nivel se sustenta en la capacidad de percepción y definición de los problemas, el conocimiento técnico necesario para articular políticas a largo plazo y las capacidades para ponerlo en práctica. Todo ello debe estar repartido entre múltiples responsables (estatales, privados y mediadores). La creación de estructuras en el mesonivel no puede estar solo activada por la política pública, sino que las empresas, las instituciones intermediarias y las asociaciones pueden y deben contribuir a la activación del desarrollo de la localidad.

En lo referente a las políticas gubernamentales para el posicionamiento y desarrollo de la región donde están asentadas las empresas objeto de esta investigación, se establece un Modelo de Desarrollo Turístico Sustentable (CORPOTULIPA, 2009) con un escenario de desarrollo a 30 años y con una estrategia de ejecución de más del $80 \%$ de las potencialidades turísticas del territorio, mediante la gestión integrada y participativa de entes públicos y privados conciliando las necesidades de la comunidad receptora, los intereses particulares con la política económica y ambiental del Estado, potenciando a Paraguaná como puerta de entrada del turismo internacional del occidente de venezuela.

El modelo de desarrollo para la Zona Libre de Paraguaná se orienta fundamentalmente en crear un clima propicio para estimular el desarrollo económico de la localidad a través del impulso del sector comercio (donde todas las personas de la localidad pueden tener participación, a través de la venta al detal de mercancías de uso personal y doméstica), y a partir de allí, promover el encadenamiento productivo en la prestación de servicios turísticos. Además, se favorece la promoción de otros sectores tales como: inmobiliario, bancario, transporte, aduaneros y demás servicios complementarios que estimulan el flujo de turistas y visitantes a la localidad.

En la práctica se evidencia que la implementación de las políticas para el desarrollo de la localidad ha traído como consecuencia la diversificación de la economía y la promoción de la actividad turística. Dentro de los resultados se observa el establecimiento de nuevos negocios, tanto con inversiones de envergadura, como en medianas y pequeñas empresas, además de la inserción de las comunidades al proceso productivo de la región a través de la creación de redes de desarrollo endógeno para los servicios turísticos.

Es significativo subrayar que todo lo antes mencionado ha originado la creación de la cadena de valor territorial a partir de las actividades turísticas y comerciales que se generan en la península y que están destinadas a satisfacer el consumo de los flujos turísticos. Al mismo tiempo, es necesario reseñar el papel de los intermediarios y organismos dependientes del estado venezolano que han impulsado la administración y operatividad del régimen fiscal de la zona libre en gestión conjunta con organismos gubernamentales, comunidades, instituciones públicas y privadas; satisfaciendo las expectativas de los inversionistas, los turistas y elevando la calidad de vida de la población local. 


\subsection{Nivel Meta}

El nivel meta supone la creación de estructuras sociales que aumentan la capacidad de los diferentes grupos de actores para articular sus intereses y hacer frente común a las exigencias de la organización técnica, social, ecológica y de la economía mundial. Para alcanzar un nivel meta adecuado deben cumplirse dos factores esenciales: por un lado la clara separación entre las instituciones del estado, la empresa privada y los organismos intermedios, y por otro, una permanente disposición al diálogo entre los actores sociales, que permita el establecimiento de consensos para aprovechar los procesos de $\mathrm{co}^{-}$ municación y aprendizaje que se den en la discusión (Esser et al., 2001, 147).

Ahora bien, cuando se consultó a los gerentes del sector objeto de estudio sobre su percepción sobre algunos aspectos vinculados con el metanivel de competitividad en su entorno se observa lo siguiente: un significativo porcentaje $(77,78 \%)$ considera que existe capacidad de organización e interacción estratégica entre los diferentes actores vinculados al turismo que hacen vida en la península. Sin embargo, un 55,55\% expresa que en bajo grado los diferentes entes e instituciones relacionadas con la actividad turística están orientados al aprendizaje colectivo y a la resolución de problemas de la localidad. Además, la disposición al diálogo entre los diferentes grupos de interés que interactúan en el desarrollo de Paraguaná como destino turístico solo es percibida de manera favorable por un $33,33 \%$ de los encuestados (Tabla 2).

$\mathrm{Al}$ respecto de los resultados antes descritos es necesario referir que los modernos enfoques de desarrollo plantean que la competitividad no se basa solo en las políticas que dejan operar al mercado, sino que es un asunto de toda la sociedad, de su capacidad de aprender y resolver problemas mediante un entramado de organizaciones e instituciones. En tal sentido, se hace necesario que los diferentes actores que interactúan en la actividad turística y comercial que se despliega en la península de Paraguaná se inscriban en una visión de conjunto que implica la conformación de estructuras sociales que permitan articular sus intereses y hacer frente común a las exigencias que impone el mercado, a través de la implementación de procesos de aprendizaje colectivo e innovación.

Tabla 2. Rasgos de Competitividad (Nivel Meta) ${ }^{4}$

\begin{tabular}{|c|c|c|c|c|}
\hline $\begin{array}{l}\text { Rasgos de Competiti- } \\
\text { vidad (Nivel Meta) }\end{array}$ & $\begin{array}{l}\text { Bajo } \\
\text { Grado }\end{array}$ & $\begin{array}{c}\text { Mediano } \\
\text { Grado }\end{array}$ & $\begin{array}{l}\text { Alto } \\
\text { Grado }\end{array}$ & NA \\
\hline \multicolumn{5}{|c|}{ (Expresado en términos porcentuales) } \\
\hline $\begin{array}{l}\text { 1. En qué nivel conside- } \\
\text { ra usted que existe dis- } \\
\text { posición al diálogo entre } \\
\text { los diferentes grupos de } \\
\text { interés que interactúan } \\
\text { en el desarrollo de Pa- } \\
\text { raguaná como destino } \\
\text { turístico }\end{array}$ & 11.11 & 55.55 & 33.33 & \\
\hline $\begin{array}{l}\text { 2. En qué medida consi- } \\
\text { dera que hay capacidad } \\
\text { de organización e inte- } \\
\text { racción estratégica en- } \\
\text { tre los diferentes actores } \\
\text { vinculados al turismo } \\
\text { que hacen vida en lo- } \\
\text { calidad }\end{array}$ & & 22.22 & 77.78 & \\
\hline $\begin{array}{l}\text { 3. En qué grado consi- } \\
\text { dera usted que los dife- } \\
\text { rentes actores relaciona- } \\
\text { dos con la actividad tu- } \\
\text { rística están orientados } \\
\text { al aprendizaje colectivo } \\
\text { y a la resolución de pro- } \\
\text { blemas de la localidad }\end{array}$ & 55.55 & 44.44 & & \\
\hline
\end{tabular}

\section{Lineamientos estratégicos para el desarrollo del sector turístico hotelero de la península de Paraguaná a partir del enfoque de competitividad sistémica}

Del análisis de los resultados obtenidos en cada uno de los niveles comprendidos en el enfoque sistémi$\mathrm{co}$, considerando como unidad de estudio el sector

4 Fuente: Elaboración propia a partir de instrumento de recolección de datos del año 2011. 
conformado por las empresas de alojamiento turístico de Paraguaná, se desprenden las siguientes propuestas estratégicas:

\subsection{Nivel Micro: Fortalecimiento de los procesos productivos clave y de apoyo para la prestación de servicios de hotelería.}

Las empresas objeto de estudio deben asumir que para ser competitivas tienen que orientarse a la búsqueda de eficiencia, calidad, flexibilidad y productividad, y para ello deben comenzar por consolidar los procesos clave o primarios, los cuales están vinculados con la prestación de servicios turísticos de hospedaje y otros colaterales o conexos. Así mismo, deben fortalecer sus actividades de apoyo que de alguna manera también inciden en la prestación de los servicios que ofrecen.

Por otra parte, es necesario destacar que tales procesos pueden ser mejorados mediante la articulación con otras organizaciones en redes de colaboración mutua, cuyo énfasis sea la innovación como factor central del desarrollo económico.

En tal sentido, las organizaciones deben activar los potenciales de aprendizaje e innovación en todas las áreas operativas de la empresa, a través de redes de cooperación, las cuales deben estar apoyadas por diversas instituciones y un contexto institucional con capacidad para fomentar la innovación.

\subsection{Nivel Meta: Conformación de Complejos Productivos para la prestación de servicios turísticos.}

Se entiende por complejo productivo una concentración sectorial y/o geográfica de empresas que se desempeñan en las mismas actividades o en actividades estrechamente relacionadas, con importantes y acumulativas economías externas, de aglomeración y especialización (por la presencia de productores, proveedores y mano de obra especializada y de servicios conexos específicos al sector) y con la posibilidad de llevar a cabo una acción conjunta en la búsqueda de eficiencia colectiva (The Cluster Competitiveness Group, 2002, 3).

Se propone para el caso objeto de estudio la conformación de estructuras formales que estén inte- gradas por las empresas que prestan servicios de alojamiento en la localidad, pero que además incluyan sectores empresariales que ofrezcan productos y servicios conexos, tales como: trasporte de viajeros, intermediarios turísticos, empresas relacionadas con la restauración, empresas proveedoras de actividades recreativas, eventos y visitas a lugares de interés turístico, entre otras. La conformación de tales complejos productivos se debe establecer de manera deliberada y consciente. Además, las empresas involucradas deben acordar las acciones a desarrollar para lograr el cambio estructural en base al desarrollo turístico de la península.

\subsection{Nivel Meso: Creación de estructuras sociales que permitan la articulación de los diferentes actores para hacer frente a los requerimientos del entorno y promuevan el dialogo entre ellos.}

La institución encargada de la gestión y el desarrollo de la península de Paraguaná como destino turístico (CORPOTULIPA) debe establecer estrategias de vinculación estrecha y continua con organismos científicos y tecnológicos (tales como universidades locales, centros de investigación formación y desarrollo, oficinas de consultorías, entre otros), instituciones financieras, organizaciones especializadas en gestión del turismo y otros entes de carácter público y privado, con el fin de incrementar y fortalecer el tejido empresarial existente, impulsando además a los nuevos inversionistas y emprendedores. Por lo tanto, la tarea de esta organización es convertirse en una especie de agencia de desarrollo local que tendría entre sus funciones la creación de estructuras sociales que permitan aumentar la capacidad de los diferentes grupos de actores involucrados en el desarrollo de la península para articular sus intereses y hacer frente común a las exigencias de la demanda turística y del entorno en general.

\subsection{Nivel Macro: Estabilización del contexto macroeconómico.}

Esta estrategia depende exclusivamente de las políticas macroeconómicas que se establezcan desde el Estado y sus diferentes órganos centrales. Es por 
ello, que la estabilización del contexto macroeconómico demanda no sólo un concepto congruente en términos tecnocráticos, sino también un esfuerzo político considerable. El éxito estará asegurado únicamente si el gobierno se muestra resuelto a imponer las difíciles y conflictivas reformas, si consigue organizar una coalición nacional de fuerzas reformadoras con miras a recobrar el equilibrio de la economía, tanto interior como exterior, y si logra captar al mismo tiempo el apoyo internacional (Esser et al., 1996), así como también de los sectores de actividad marcados por las ventajas competitivas en la región.

El Mapa Conceptual que se presenta en la Ilustración 1 muestra la trama relacional de los lineamientos estratégicos expuestos en este último apartado, como un aporte por configurar una propuesta de gestión de desarrollo económico local enmarcada en el enfoque de competitividad sistémica.

\section{Conclusiones}

La competitividad sistémica se constituye en un enfoque teórico de referencia para visualizar que un sector de actividad no podrá ser competitivo por sí mismo si no cuenta con el apoyo de un nivel macro y meta donde se examinen la capacidad de la sociedad y del Estado para integrar la estrategia de crecimiento del sector, un nivel meso donde se estudia la formación de un entorno capaz de fomentar el crecimiento, y un nivel micro que haga énfasis en la eficiencia, calidad, flexibilidad y productividad basada en redes de cooperación entre las empresas del sector y los actores involucrados. Partiendo de esta premisa se presentan las siguientes consideraciones finales en el marco del análisis del estudio realizado:

En el caso del sector hotelero ubicado en la península de Paraguaná se evidencia que hay un nivel micro que debe emprender esfuerzos de manera sostenida para mejorar la prestación de sus servicios, incrementar sus niveles de eficiencia, productividad y calidad, y por consiguiente lograr ventajas competitivas.

En cuanto al nivel macro es necesario que el Estado formule políticas económicas que propendan al crecimiento y la estabilidad sobre la base de sistemas fiscales sólidos que proporcionen los recur-

Ilustración 1. Trama relacional de lineamientos estratégicos como propuesta de gestión del desarrollo local de la Península de Paraguaná sobre la base del sector empresarial turístico hotelero ${ }^{10}$



10 Fuente: Elaboración propia 
sos adecuados para el desempeño de las funciones públicas, un tipo de cambio competitivo, tasas de interés reales y un desarrollo financiero sostenido. En tal sentido, en el caso venezolano se requiere la implementación de profundos cambios en materia macroeconómica para superar el impacto de las medidas gubernamentales aplicadas durante los últimos años.

En lo correspondiente al meso nivel es conveniente destacar que se han realizado algunos intentos de conformar estructuras sociales de carácter local con actores públicos, privados y la comunidad en el contexto del desarrollo de la península de Paraguaná como destino turístico emergente.

En el meta nivel también se evidencia la disposición al diálogo e interacción estratégica entre las empresas que conforman el sector objeto de esta indagación, con el fin de mejorar su capacidad de aprendizaje y resolver problemas mediante un entramado de organizaciones e instituciones fundamentadas en el proyecto de desarrollo turístico local.

Ahora bien, para lograr el desarrollo competitivo del sector hotelero de Paraguaná a través de la interacción de los diferentes elementos que componen los niveles analíticos del enfoque de competitividad sistémica y producto del análisis realizado en este artículo se proponen las siguientes líneas estratégicas generales: fortalecimiento de los procesos productivos clave y de apoyo de las empresas hoteleras para la prestación del servicio; conformación de complejos productivos para la prestación de servicios vinculados con la demanda de los flujos turísticos; creación de estructuras sociales que permitan la articulación de los diferentes actores para hacer frente a los requerimientos del entorno y promuevan el dialogo entre ellos; y estabilización del contexto macroeconómico, la cual depende exclusivamente del Estado y sus órganos centrales.

El éxito de esta propuesta estratégica dependerá de la interacción compleja, amplia y concertada de todos los actores involucrados para promover el desarrollo económico local de la península y del sector turístico en su conjunto.

\section{Referencias}

Antonorsi, M. (1999). Guía Práctica de la Empresa Competitiva. Venezuela: Ediciones Venezuela Competitiva.

Comisión Económica para la América Latina y el Caribe (CEPAL) (2000). Equidad, desarrollo y ciudadanía. En: Publicación de las Naciones Unidas. LC/G.2138-P.

Comisión Económica para la América Latina y el Caribe (CEPAL) (2001). Elementos de Competitividad Sistémica de las Pequeñas y Medianas Empresas (PYME) del Istmo Centroamericano. En: Naciones Unidas. Comisión Económica para la América Latina y el Caribe. Disponible en: http://www.eclac.org/ publicaciones/xml/1/9481/1499.pdf

Comisión Económica para la América Latina y el Caribe (CEPAL) (2003). Globalización y Desarrollo. Una reflexión desde América Latina y el Caribe. Bogotá: Banco Mundial y Alfaomega Colombiana S.A.

Consejo Nacional de Promoción de Inversiones (CONAPRI) (2008). Corpotulipa recibió la Certificación ISO 9001:2000. Disponible: http://www.conapri.org/ArticleDetailIV.asp?arti cleid $=322703 \&$ Categoryld $2=23437$

Corporación para la Zona Libre para el Fomento de la Inversión Turística en la Península de Paraguaná (CORPOTULIPA, 2009). Zonal Libre de Paraguaná como instrumento del Estado para alcanzar un modelo productivo de beneficio social.

Esser, K., Hillebrand, W., Messner D. \& Meyer-Stamer, J. (1996). Competitividad Sistémica: Nuevo desafíos a las empresas y a la política. En: Revista de la CEPAL, No. 59:39 - 52.

Esser, K., Hillebrand, W., Messner D. \& Meyer-Stamer, J. (2001). Competitividad sistémica y desarrollo. En: Thiel, R. (Editor). Teorías del desarrollo. Nuevos enfoques y problemas. Caracas: Editorial Nueva Sociedad.

Fea, U. (1995). Competitividad es calidad total. Colombia: Ediciones Alfaomega, S.A.

Fernández, L. (1974). Teoría y técnica del turismo. Madrid: Editora Nacional.

Ferrer, J. \& Vásquez, J. (2005). La competitividad microsistémica como estrategia empresarial para el desarrollo de la pequeña y mediana empresa zuliana. En: Desarrollo Económico Regional en Venezuela. Foros, 11:155-180.

Francés, A. (2003). Turismo: competitividad y estrategia. Planes de desarrollo de destinos turísticos con el Cuadro de Mando Integral. Caracas: IESA.

Ley de Zona Libre para el Fomento de la Inversión Turística en la Península de Paraguaná (1998). Gaceta Oficial de la República Bolivariana de Venezuela. Decreto No 36.517. (14 de Agosto de 1998).

Madoery, O. (2000). El valor de la Política de Desarrollo Local. Disponible en: http://www.cedet.edu.ar/Archivos/Bibliotecas/madoery.pdf

McIntosh, R., Goeldner, C. \& Ritchie, J.R. (2008). Turismo. Planeación, Administración y Perspectivas. México D.F: Editorial Limusa, S.A.

Méndez, C. (2001). Metodología. Diseño y Desarrollo del Proceso de Investigación. Madrid: McGrawHill Interamericana de España, S.A.

Mochón, F. (2004). Economía y Turismo. Madrid: McGraw-Hill/ Interamericana de España, S.A.U.

Morales, M. \& Castellanos, O. (2007). "Estrategias para el fortale- 
cimiento de las Pyme de base tecnológica a partir del enfoque de competitividad sistémica". En: Innovar, 17(29): 115-136.

Narváez, M., Fernández, G. \& Senior, A. (2008). "El desarrollo local sobre la base de la Asociatividad Empresarial: Una propuesta estratégica”. En: Revista Opción. Universidad del Zulia, № 57:74-92.

Penfold, M., Vainrub, R. \& Dohnert, S. (2009). Volar alto: ¿Cómo enfrentan las empresas venezolanas la adversidad? En: Penfold, M. \& Vainrub, R. (Editores). Estrategias en tiempos de turbulencia. Las empresas venezolanas. Caracas: Ediciones IESA.
Ramírez, C. (2007). Hoteles. Gerencia, seguridad y mantenimiento. México: Editorial Trillas.

Rojas, P., Romero, S. \& Sepúlveda, S. (2000). Algunos ejemplos de cómo medir la competitividad. Cuaderno Técnico № 14 . San José Costa Rica: IICA.

The Cluster Competitiveness Group (2002). “What is a cluster? Cluster-based policies”. Disponible en: http://www.competitiveness.com

Villamediana, Z. \& Berbesi, L. (2009). Creciendo con el Turismo. Caracas: FUNDES. 